Z. Klin. Chem. Klin. Biochem.

11. Jg. 1973 , S. $311-318$

\title{
Homotrope kooperative Effekte und aufsteigende B/F-Kurven bei Hormon-Antikörperreaktionen
}

\author{
Von H.-J. Bartels und R.-D. Hesch \\ Technische Assistenz F. Presche \\ Mediqiniscbe Universitätsklinik Göttingen, Arbeitsgruppe Endokrinologie
}

(Eingegangen am 28. November 1972/12. März 1973)

Insofern sich die Sätze der Mathematik auf die Wirklichkeit beziehen, sind sie nicht sicher, und insofern sie sicher sind, beziehen sie sich nicht auf die Wirklichkeit.

Albert Einstein

Faßt man Antikörper als allosterische Proteine, Hormone als die zugehörigen Liganden auf, so etgeben homotrope, kooperative allosterische Effekte aufsteigende B/F-Kurven. Die formelmäßige Beschreibung der Hormon-Antikörperreaktion folgt dabei dem Modell von Monod-WyMan-Changeux.

Die zentrale Gleichung des von uns in einer früheren Arbeit empirisch gefundenen stark vereinfachten Modells aufsteigender B/F-Kurven stellt sich als Spezialfall einer bereits 1910 von HrLl angegebenen Formel für die sigmoidalen Kurven bei der Bindung von Sauerstoff an Hämoglobin dar. Bei der quantitativen Beschreibung von Hormon-Antikörperreaktionen hat man die Heterogenität der Antikörperpopulationen zu berücksichtigen. Es wird gezeigt: Heterogenität und Kooperativität können sich in der Weise überlagern, daß trotz homotroper Effekte ein SCATCHARD-plot linear abfallende B/F-Kurven ergibt.

\section{Homotropic cooperative effects and rising B/F-curves in hormone-antibody reactions}

If antibodies are considered as allosteric proteins, and hormones as the corresponding ligands, then homotropic, cooperative allosteric effects are represented by rising $B / F$ curves. The formalized description of the hormone-antibody reaction thus agrees with the model of MONOD, WyMAN and ChangeuX.

In an earlier publication we derived empirically a greatly simplified model for rising B/F curves. The central equation of this simplified model represents a special case of the formula for the sigmoidal curves for the binding of oxygen to haemoglobin, which was reported by HILL in 1910. In the quantitative study of hormone - antibody reactions, the heterogeneity of the antibody populations must be taken into account. It is shown that heterogeneity and cooperative activity can overlap, so that a SCATCHARD plot can give linearly decreasing $\mathrm{B} / \mathrm{F}$ curves, in spite of homotropic effects.

Die Frage einer quantitativen Beschreibung der von BOHR 1903 beobachteten sigmoidalen $\mathrm{O}_{2}$-Dissoziationskurven bei der Bindung von Sauerstoff an Hämoglobin war jahrzehntelang Gegenstand ausführlicher Untersuchungen. Die von HILl 1910 empirisch gefundene Beziehung

$$
\mathrm{Y}=\frac{\mathrm{K} \cdot \mathrm{p}^{\mathrm{n}}}{1+K \mathrm{p}^{\mathrm{n}}}(\mathrm{n} \approx 2,6) \text {, }
$$

die formal einem Gleichgewicht $\mathrm{Hb}_{n}+\mathrm{nO}_{2} \rightleftharpoons$ $\mathrm{Hb}_{\mathbf{n}}\left(\mathrm{O}_{2}\right)_{\mathrm{n}}$ entspricht, konnte in guter Annäherung die experimentellen Daten beschreiben. Die gleiche Frage ist später neben anderen von AdaIR (1925), Pauling (1935) und sehr viel später nach einer genaueren Strukturaufklärung des Hämoglobinmoleküls durch Perutz et al. (1960) von Monod-Wyman-Changeux (1964) und in anderer Weise von KoshLAND-NEḾETHYYFilmer (1966) diskutiert worden. Die Annahme einer indirekten Beeinflussung von verschiedenen Bindungsstellen eines Proteins gegen einen oder verschiedene Liganden z. B. bei Enzym-Substrat-Reaktionen, die Annahme von Konformationsänderungen bei Enzymen, die sich aus mehreren Untereinheiten zusammensetzen, ist inzwischen in vielen Versuchssystemen experimentell gut bestätigt $(1,2)$. Der kooperative Effekt bei der $\mathrm{O}_{2}-$ Bindung an Hämoglobin erscheint inzwischen nicht mehr als vereinzeltes Phänomen, sondern eher als ein Fall von vielen in einer sehr allgemeinen Situation (2).

Im Zusammenhang mit der radioimmunchemischen Bestimmungsmethode von ACTH beschrieben MATsuKurA et al. 1971 einen kooperativen Effekt bei der Bindung von ACTH an spezifische Antikörper (3). Die Kooperativität ergibt hier aufsteigende B/F-Kurven. Das Phänomen aufsteigender B/F-Kurven erscheint uns aufgrund eigener Untersuchungen (4) als ein spezieller Fall einer sehr allgemeinen Situation:

Aufsteigende B/F-Kurven sind inzwischen auch bei anderen Hormon-Antikörperreaktionen nachgewiesen, nämlich außer beim ACTH auch noch bei folgenden Hormonen: beim Trijodthyronin $\left(\mathrm{T}_{3}\right)$, beim menschlichen Calcitonin (HCT), beim Parathormon (PTH), bei dem humanen Placental Lactogen (HPL), beim Gastrin, beim thyreotropen Hormon (TSH) und beim mensch- 
lichen Choriongonadotropin (HCG) (5), die Frage einer theoretischen Behandlung dieses Phänomens ist noch nicht ausdiskutiert $\left.{ }^{\mathrm{l}}\right)$.

Eine erste quantitative Beschreibung aufsteigender B/F-Kurven bei Antiseren gegen Trijodthyronin ist kürzlich von uns gegeben worden (4). Dabei gingen wir von einem Gleichgewicht der Form

$2 \mathrm{H}+\mathrm{AK} \rightleftharpoons \mathrm{H}_{2} \mathrm{AK}$

$$
\mathrm{K}[\mathrm{h}]^{2}\left([\mathrm{AK}]-\left[\mathrm{h}_{2} \mathrm{ak}\right]\right)=\left[\mathrm{h}_{2} \mathrm{ak}\right]
$$

aus ${ }^{2}$.

Mit $B=2\left[h_{2} a k\right]$ und $F=[h]$ hat man dann wegen Gleichung 3:

$$
\frac{\mathrm{B}}{2[\mathrm{AK}]}=\frac{\mathrm{K} \cdot \mathrm{F}^{2}}{1+\mathrm{K} \cdot \mathrm{F}^{2}} \text {. }
$$

Dies entspricht genau der von HILL 1910 angegebenen quantitativen Beziehung in Gleichung 1 mit $\mathrm{n}=2$. Es liegt jetzt nahe zu fragen, inwieweit sich die quantitative Beschreibung von Hormon-Antikörperreaktionen einem allgemeinen Schema unterordnet, ob man z. B. das Modell von Monod-WyMan-Changeux auf diese Situation anwenden kann. Dies Modell beschreibt die experimentellen Daten bei der Bindung von Sauerstoff an Hämoglobin besser, als das mit Gleichung 1 möglich ist.

In der vorliegenden Arbeit wird noch einmal kurz die klassische Situation bivalenter Antikörper und monovalenter Antigene unter der Annahme diskutiert, daß sich die $z$ wei Bindungsstellen weder direkt noch indirekt beeinflussen. Danach wird die qualitative Gestalt der theoretischen B/F-Standardkurven untersucht, die bei Anwendung des Monodschen Modells zu erwarten sind, wenn man eine allosterische Konformationsänderung der Antikörpermoleküle voraussetzt, die bei Bindung eines Hormonmoleküls stabilisiert wird.

\section{Mehrfache Gleichgewichte} bei Hormon-Antikörperreaktionen

Geht man bei der Hormon-Antikörperreaktion von zwei gekoppelten Gleichgewichten

(Gl. 5)

(Gl. 6)

$$
\begin{aligned}
\mathrm{H}+\mathrm{AK} & \rightleftharpoons \mathrm{HAK} \\
\mathrm{H}+\mathrm{HAK} & \rightleftharpoons \mathrm{H}_{2} \mathrm{AK}
\end{aligned}
$$

aus, so hat man nach dem Massenwirkungsgesetz

$$
\text { (Gl. 7) } \quad[\mathrm{hak}]=\mathrm{K}_{1}[\mathrm{~h}][\mathrm{ak}]
$$$$
\left[\mathrm{h}_{2} \mathrm{ak}\right]=\mathrm{K}_{2}[\mathrm{~h}][\mathrm{hak}]
$$

mit Affinitätskonstanten $\mathrm{K}_{1}$ und $\mathrm{K}_{2},[\mathrm{~h}]$, [ak], [hak] und $\left[h_{2} a k\right]$ bezeichnen dabei die jeweils beim Gleichgewicht vorhandenen Konzentrationen. Setzt man voraus - und das ist wegen der Symmetrie der Antikörpermoleküle eigentlich naheliegend -, daß die Gleichgewichtskonstanten beider Bindungsstellen gleich sind, und daß keine gegenseitige Beeinflussung stattfindet, so hat man bei Berücksichtigung des statistischen Fak-

\footnotetext{
1) Vgl. Beittäge von B. D. Weintraub und D. Rodbard, IV. Int. Congr. of Endocrinology, Washington, June 1972, sowie: Weintraub, B. D., Rosen, S. W., McCammon, J. A. \& .PerlMAN, R. L.: (1973), Endocrinology im Druck.

2) Abkürzungen siehe Anhang.
}

tors $4=\mathrm{K}_{1} / \mathrm{K}_{2}$ (6) mit $\mathrm{K}_{1}=2 \mathrm{~K}$ und den Gleichungen 7 und 8:

(Gl. 9)

(Gl. 10)

$$
\begin{aligned}
{[\mathrm{hak}] } & =2 \mathrm{~K}[\mathrm{~h}][\mathrm{ak}] \\
{\left[\mathrm{h}_{\mathbf{2}} \mathrm{ak}[\right.} & =\mathrm{K}^{2} \cdot[\mathrm{h}]^{2}[\mathrm{ak}] .
\end{aligned}
$$

Bezeichnen jetzt $[\mathrm{H}]$ und $[\mathrm{AK}]$ die jeweiligen Ausgangskonzentrationen vor dem Gleichgewicht und setzt man weiter

$$
\begin{aligned}
& \mathbf{F}=[\mathrm{h}] \\
& \mathbf{B}=2\left[\mathrm{~h}_{2} \mathrm{ak}\right]+[\mathrm{hak}],
\end{aligned}
$$

so hat man:

$$
\mathrm{B} / \mathrm{F}=\frac{2\left[\mathrm{~h}_{2} \mathrm{ak}\right]+[\mathrm{hak}]}{[\mathrm{h}]}=\frac{2\left[\mathrm{~h}_{2} \mathrm{ak}\right]+[\mathrm{hak}]}{[\mathrm{H}]-2\left[\mathrm{~h}_{2} \mathrm{ak}\right]-[\mathrm{hak}]} .
$$

Es folgt

(Gl. 11)

$$
2\left[\mathrm{~h}_{2} \mathrm{ak}\right]+[\mathrm{hak}]=[\mathrm{H}] \frac{\mathrm{B} / \mathrm{F}}{1+\mathrm{B} / \mathrm{F}} .
$$

Weiter ist dann:

$$
\text { (Gl. 12) }[\mathrm{h}]=[\mathrm{H}]-[\mathrm{H}] \frac{\mathrm{B} / \mathrm{F}}{1+\mathrm{B} / \mathrm{F}}=\frac{[\mathrm{H}]}{1+\mathrm{B} / \mathrm{F}} \text {. }
$$

Mit Gleichung 9 und Gleichung 10 hat man dànn weiter:

$$
\begin{gathered}
\mathrm{B} / \mathrm{F}=2 \mathrm{~K}[\mathrm{ak}]+2 \mathrm{~K}^{2}[\mathrm{~h}][\mathrm{ak}]= \\
2 \mathrm{~K}[\mathrm{ak}]\left(1+\mathrm{K} \frac{[\mathrm{H}]}{1+\mathrm{B} / \mathrm{F}}\right) .
\end{gathered}
$$

$\mathrm{Um} \mathrm{B/F}$ als Funktion von $[\mathrm{H}]$ zu kennen, hat man in der letzten Gleichung nur noch [ak] als Funktion der Anfangskonzentration $[\mathrm{H}]$ und des Quotienten $\mathrm{B} / \mathrm{F}$ auszudrücken. Mit der Gleichung 9 ergibt sich dann nach elementaren Umformungen und mit den Abkürzungen $\mathrm{R}=\mathrm{B} / \mathrm{F}$ und $\mathrm{x}=[\mathrm{H}]$ :

(Gl. 14) $R^{2}+R(1+K x-2 K[A K])-2 K[A K]=0$.

Geht man anstelle von Gleichung 5 und Gleichung 6 von einem einfachen Gleichgewicht

$$
\mathrm{H}+\mathrm{AK} \rightleftharpoons \mathrm{HAK}
$$

$\mathrm{K}[\mathrm{h}][\mathrm{ak}]=[\mathrm{hak}]$ und monovalenten Antikörpern aus, so erhält man anstelle von Gleichung 14 die bekannte Hyperbelgleichung

(G1. 15) $\dot{\mathrm{R}}^{2}+\mathrm{R}(1+\mathrm{Kx}-\mathrm{K}[\mathrm{AK}])-\mathrm{K}[\mathrm{AK}]=0$ (1.c. 7$)$.

Gleichung 14 beschreibt B/F als Funktion von der eingesetzten Hormonkonzentration $[\mathrm{H}]$ wie Gleichung 15 als abfallende Hyperbel, aber mit anderen konstanten Parametern. Die Symmetrie der Bindungsverhältnisse stillschweigend vorausgesetzt, ergibt sich aus dem eben Gezeigten, daß ein bivalentes Verhalten der Antikörpermoleküle allein noch keine aufsteigenden $\mathrm{B} / \mathrm{F}$ Kurven hervorruft. Wenn - wie früher gezeigt (1. c. 4) - aus einem Gleichgewicht

$$
2 \mathrm{H}+\mathrm{AK} \rightleftharpoons \mathrm{H}_{2} \mathrm{AK}
$$

aufsteigende B/F-Kurven resultieren, so liegt dies wesentlich daran, daß sich die Glèichgewichte

$$
\mathrm{H}+\mathrm{AK} \rightleftharpoons \mathrm{HAK}
$$

$\mathrm{H}+\mathrm{HAK} \rightleftharpoons \mathrm{H}_{2} \mathrm{AK}$ mehr nach $\mathrm{H}_{2} \mathrm{AK}$ verlagern, mehr als das in der klassischen Situation statistisch zu erwarten ist. Die Vernachlässigung der Komplexe HAK ermöglicht zwar eine erste formelmäßige Be- 
schreibung der experimentellen Daten, liefert aber auf der anderen Seite noch keine befriedigende Erklärung aufsteigender B/F-Kurven.

Es liegt nahe, die Möglichkeit zu diskutieren, daß ein ausgeprägter kooperativer Effekt die Bindung der zwei Hormonmoleküle an den Antikörper begleitet und in erster Näherung die Vernachlässigung der Komplexe HAK erlaubt.

\section{Das Symmetriemodell bei Hormon-Antikörper- reaktionen}

Macht man Konformationsänderungen der Antikörpermoleküle für den kooperativen Effekt bei der Bindung der Hormonmoleküle verantwortlich, so kann man für die Beschreibung des Bindungsverhaltens auf zwei Modelle zurückgreifen: sowohl das Monodsche als auch das KosHLANDsche Modell können kooperative Effekte quantitativ beschreiben.

In dieser Arbeit wird nur die Anwendbarkeit des MoNodschen Modells (2) überprüft. Dies hat zwei Gründe:

1. Die bei dem Studium des Gleichgewichts erhaltenen Versuchsdaten ermöglichen innerhalb der experimentellen Fehlergrenzen keine Unterscheidung beider Modelle $(8,9)$.

2. Die mathematische Einfachheit des MonoD-Modells.

Um Unklarheiten bezüglich der Terminologie und der benutzten Abkürzungen zu vermeiden, werden hier kurz die wesentlichen Hypothesen und Aussagen des Modells zusammengestellt, dabei können wir uns allerdings auf unsere spezielle Situation, d. h. auf Dimere beschränken.

Das allosterische Modell postuliert zwei Konformationen des betreffenden Oligomers, die sozusagen in einem Prääquilibrium auch in Abwesenheit der Liganden vorliegen. Die beiden Konformationen zeichnen sich durch unterschiedliche Affinitäten gegenüber den Liganden aus. Weitere Postulate sind: die Symmetrie des allosterischen Proteinmoleküls in beiden Zuständen und ein "Alles- oder Nichts"-Gesetz in dem folgenden Sinn: die Untereinheiten des allosterischen Moleküls, die "Protomere“ haben innerhalb eines Moleküls die gleiche Konfiguration, man geht also von gleichzeitigen Konformationsänderungen der identischen Untereinheiten aus.

Nach dem Porterschen Strukturmodell für Antikörper vom Typ IgG (10) bestehen in unserer speziellen Situation die zwei identischen Untereinheiten aus je einer $\mathrm{H}$ - und einer L-Kette, beide Untereinheiten sind spiegelsymmetrisch zu einer Ebene angeordnet und werden durch Disulfidbrücken zusammengehalten.

Bezeichnen $R_{0}$ und $T_{0}$ die beiden postulierten Konfigurationen der Antikörpermoleküle, $L$ die Gleichgewichtskonstante für das Gleichgewicht $R_{0} \rightleftharpoons T_{0}$, $K_{R}$ und $K_{T}$ die Dissoziationskonstanten der HormonAntikörperbindung in dem R- bzw. T-Zustand, so reagieren Hormon- und Antikörpermoleküle nach folgendem Schema:

$$
\begin{gathered}
\mathrm{R}_{0} \rightleftharpoons \mathrm{T}_{0} \\
\mathrm{R}_{0}+\mathrm{H} \rightleftharpoons \mathrm{R}_{1} \quad \mathrm{~T}_{0}+\mathrm{H} \rightleftharpoons \mathrm{T}_{1} \\
\mathrm{R}_{1}+\mathrm{H} \rightleftharpoons \mathrm{R}_{2} \quad \mathrm{~T}_{1}+\mathrm{H} \rightleftharpoons \mathrm{T}_{2} .
\end{gathered}
$$

Unter Berücksichtigung der statistischen Faktoren für die verschiedenen Dissoziationskonstanten hat man dann:

$$
\begin{aligned}
& \mathrm{T}_{0}=\mathrm{LR}_{0} \\
& \mathrm{R}_{1}=\mathrm{R}_{0} 2 \frac{[\mathrm{h}]}{\mathrm{K}_{\mathrm{R}}} \quad \mathrm{T}_{1}=\mathrm{T}_{0} 2 \frac{[\mathrm{h}]}{\mathrm{K}_{\mathrm{T}}} \\
& R_{2}=R_{1} \frac{1[\mathrm{~h}]}{2 \mathrm{~K}_{\mathrm{R}}} \quad \mathrm{T}_{2}=\mathrm{T}_{1} \frac{1[\mathrm{~h}]}{2 \mathrm{~K}_{\mathrm{T}}} .
\end{aligned}
$$

Mit

$$
[\mathrm{h}]=\mathrm{F}, \mathrm{c}=\frac{\mathrm{K}_{\mathrm{R}}}{\mathrm{K}_{\mathrm{T}}}, \alpha=\frac{[\mathrm{h}]}{\mathrm{K}_{\mathrm{R}}}
$$

und

$$
\begin{gathered}
B=R_{1}+2 R_{2}+T_{1}+2 T_{2}, \\
{[A K]=R_{0}+R_{1}+R_{2}+T_{0}+T_{1}+T_{2}}
\end{gathered}
$$

hat man dann:

(Gl. 16) $\frac{\mathrm{B}}{\mathrm{F}}=\frac{1}{\mathrm{~K}_{\mathbf{R}}} \cdot 2[\mathrm{AK}] \frac{(1+\alpha)+\mathrm{Lc}(1+\mathrm{c} \alpha)}{(1+\alpha)^{2}+\mathrm{L}(1+\mathrm{c} \alpha)^{2}}$.

Es wird hier gezeigt, daß sich bei stark ausgeprägten homotropen allosterischen Effekten, d. h. für großes L und sehr kleines $c$ aufsteigende B/F-Kurven ergeben

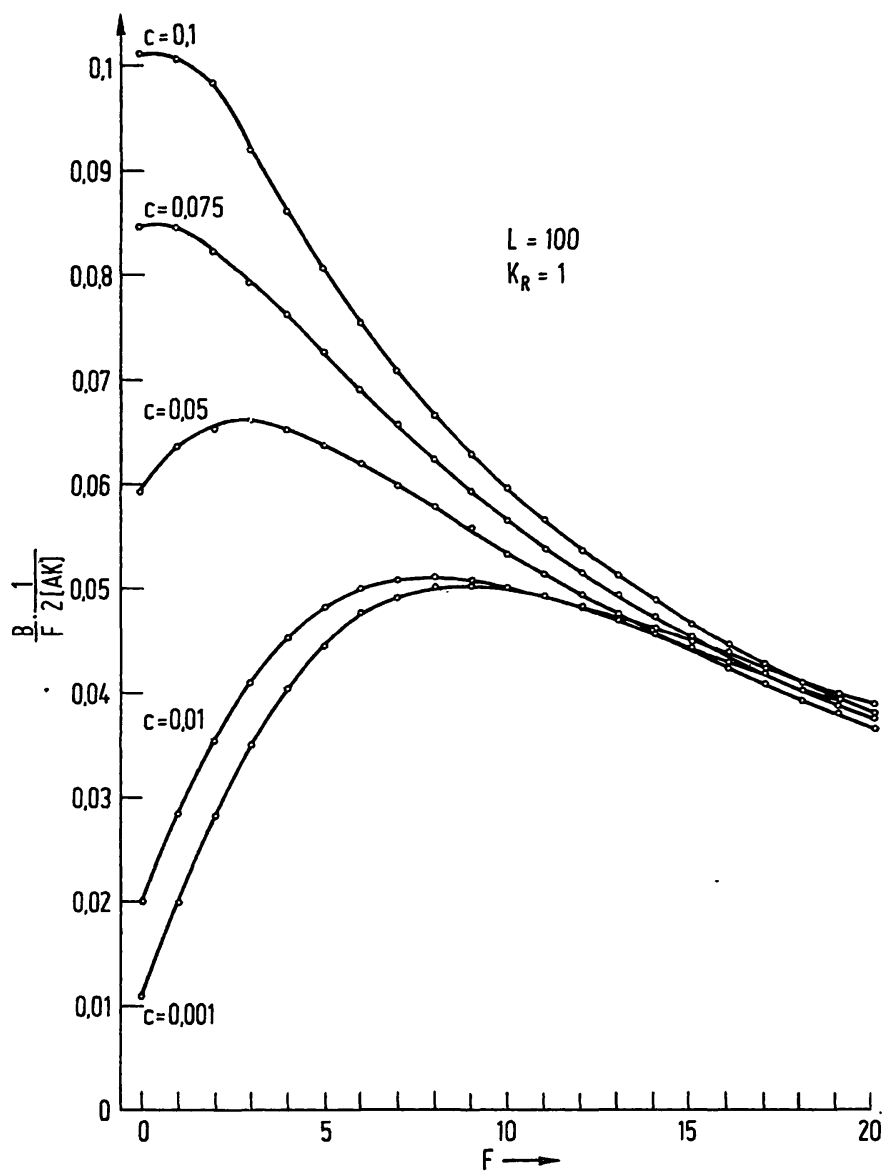

Abb. 1

Nach Gleichung 16 tabellierte B/F-Kurven. Das qualitative Anfangsverhalten wird wesentlich von dem Grad der Kooperativität bestimmt. 
(Abb. 1). Bezeichnet $[\mathrm{H}]$ die Gesamthormonkonzentration, so ist

$$
[\mathrm{H}]=[\mathrm{h}]+\mathrm{B}=\mathrm{K}_{\mathrm{n}} \cdot \alpha+2[\mathrm{AK}] \frac{\alpha(1+\alpha)+\mathrm{L} \alpha \mathrm{c}(1+\mathrm{c} \alpha)}{(1+\alpha)^{2}+\mathrm{L}(1+\mathrm{c} \alpha)^{2}} .
$$

Wegen

$$
\frac{\mathrm{d}}{\mathrm{d} \alpha}\left(\frac{\alpha(1+\alpha)+\operatorname{L} \alpha c(1+c \alpha)}{(1+\alpha)^{2}+\mathrm{L}(1+c \alpha)^{2}}\right) \geqq 0 \quad \text { ist }
$$

zu studieren. $\mathbf{B} / \mathbf{F}$ als Funktion von $[\mathrm{H}]$ wird genau dann einen aufsteigenden Ast haben, wenn dies für $\mathrm{B} / \mathrm{F}$ als Funktion von $\alpha$ der Fall ist. Mit der Bestimmung der Lage des Maximums wird gleichzeitig die Existenz $\frac{\mathrm{d}[\mathrm{H}]}{\mathrm{d} a}>0$ und $[\mathrm{H}]$ ist streng monoton wachsende des aufsteigenden Astes nachgewiesen. Bezeichnet $\alpha_{\text {MaX }}$ den $\alpha$-Wert mit maximalem B/F bei fester Antikörperkonzentration, so ergibt sich $[\mathrm{H}]_{M \Lambda X}$ aus Funktion von $\alpha$ und umgekehrt. Um das qualitative. suchen, genügt es daher, $\mathrm{B} / \mathrm{F}$ in Abhängigkeit von $\alpha$

Gleichung 17. Es ist nun

$$
\frac{\mathrm{d}(\mathrm{B} / \mathrm{F})}{\mathrm{d} \alpha}=\frac{1}{\mathrm{~K}_{\mathrm{R}}} \cdot 2[\mathrm{AK}] \cdot \frac{\left((1+\alpha)^{2}+\mathrm{L}(1+\mathrm{c} \alpha)^{2}\right)\left(1+\mathrm{Lc}^{2}\right)-((1+\alpha)+\mathrm{Lc}(1+\mathrm{c} \alpha))(2(1+\alpha)+2 \mathrm{Lc}(1+\mathrm{c} \alpha))}{\left((1+\alpha)^{2}+\mathrm{L}(1+\mathrm{c} \alpha)^{2}\right)^{2}}
$$

und daher ist $\frac{d(B / F)}{d \alpha} \gtreqless 0$, je nachdem

$$
\left((1+\alpha)^{2}+\mathrm{L}(1+\mathrm{c} \alpha)^{2}\right)\left(1+\mathrm{Lc}^{2}\right) \gtreqless((1+\alpha)+(\mathrm{Lc}(1+\mathrm{c} \alpha))((2(1+\alpha)+2 \mathrm{Lc}(1+\mathrm{c} \alpha))
$$

ist und das ist äquivalent der Bedingung:

$$
\alpha^{2}+2 \alpha \frac{\left(1+L c^{2}+L c+L^{2} c^{3}\right)}{\left(1+2 L c^{2}+L^{2} c^{4}\right)}-\frac{L+L c^{2}-L^{2} c^{2}-4 L c}{1+2 L c^{2}+L^{2} c^{4}} \lesseqgtr 0 .
$$

Für kleine c-Werte und großes $L$ gibt es genau ein $\alpha_{\text {MAX }} \geqq 0$ mit $\frac{\mathrm{d}(\mathrm{B} / \mathrm{F})}{\mathrm{d} \alpha}\left(\alpha_{\mathrm{MAX}}\right)=0$, es ist

$$
\alpha_{\operatorname{MAX}}=-\frac{1+L c^{2}+L c+L^{2} c^{3}}{1+2 L c^{2}+L^{2} c^{4}}+\sqrt{\left(\frac{1+L c^{2}+L c+L^{2} c^{3}}{1+2 L c^{2}+L^{2} c^{4}}\right)^{2}+\frac{L+L c^{2}-L^{2} c^{2}-4 L c}{1+2 L c^{2}+L^{2} c^{4}}} .
$$

Für den maximalen $B / F-W e r t ~(B / F)_{\text {MAX }}$ gilt dann (Gl. 19)

$$
(\mathrm{B} / \mathrm{F})_{\mathrm{MAX}}=\frac{1}{\mathrm{~K}_{\mathrm{R}}} \cdot[\mathrm{AK}] \frac{1+\mathrm{Lc}^{2}}{1+\alpha_{\mathrm{MAX}}+\mathrm{Lc}\left(1+\mathrm{c} \alpha_{\mathrm{MAX}}\right)} \text {. }
$$

Da nach Gleichung $18 \alpha_{M \Delta X}$ nur von $L$ und $\mathrm{c}$ abhängt, hat man:

$(\mathrm{B} / \mathrm{F})_{\mathrm{MAAX}}$ wächst linear mit steigender Antikörperkonzentration.

Nach Gleichung 17 wächst auch die Konzentration $[\mathrm{H}]_{\text {MAX }}$, die den maximalen (B/F)-Wert ergibt, linear mit der eingesetzten Antikörperkonzentration.

\section{Heterogenität der Antikörper}

Die Untersuchung des Bindungsverhaltens und der Bindungsstellen von Antikörpermolekülen wird durch die ausgeprägte Heterogenität der meisten Antikörperpopulationen erschwert (11). So berichteten NrsonOFF und Pressman von gekrümmten Kurven bei der Auftragung der Gleichgewichtsdaten bei der Bindung von $p$-Jodobenzoat an Anti- $p$-azobenzoat-antikörper und $z$ war bei der Auftragung von $1 / F$ gegenüber $1 / B$, die Bindungskurve krümmt sich zur 1/F-Achse. Dieses Bindungsverhalten kann durch die bekannte SIPsGleichung beschrieben und mit der Heterogenität der Bindungsaffinitäten verschiedener Antikörpermoleküle erklärt werden.

Ein solches "Krümmungsverhalten" ist dem von uns kürzlich bei der Bindung von radioaktiv markiertem Trijodthyronin $\left(\mathrm{T}_{3}\right)$ an spezifische Antikörper gegen $\mathrm{T}_{3}$ beobachteten genau entgegengesetzt. Die Vermutung liegt nahe, daß Heterogenität und kooperative Effekte bei Antikörpern sich antagonistisch überlagern (12), unter Umständen sogar eine lineare Abhängigkeit von 1/F gegenüber $1 / \mathrm{B}$ ergeben, ohne daß die HormonAntikörperreaktion nach dem einfachen Schema:

$$
\mathrm{H}+\mathrm{AK} \rightleftharpoons \mathrm{HAK}
$$

abläuft. Dieser Überlagerungseffekt von Heterogenität und Kooperativität soll jetzt quantitativ diskutiert werden. Die Heterogenität der Antikörpermoleküle wird dabei nach Srps (13) durch eine zu einem Mittelwert $K_{0}$ symmetrische Verteilungsfunktion $f(K)$ für die Affinitätskonstanten $\mathrm{K}$ der einzelnen Antikörpermoleküle beschrieben und die kooperative Bindung der Hormonmoleküle an die bivalenten Antikörpermoleküle folgt in erster vereinfachter Näherung dem in 1. c. (4) benutzten Schema von Gleichung 2, welches die Komplexe HAK vollständig vernachlässigt. In einem infinitesimalen Bereich ist dann der Quotient aus der Anzahl der Bindungsstellen mit einer bestimmten Affinitätskonstanten $\mathrm{K} \mathrm{zu}$ der Gesámtanzahl der überhaupt verfügbaren Bindungsstelleñ der Antikörpermoleküle durch

gegeben, oder:

$$
\frac{\mathrm{dN}}{\mathrm{N}}=\mathrm{f}(\mathrm{K}) \mathrm{dK}
$$

$$
\frac{d(2[a k])+d\left(2\left[h_{2} a k\right]\right)}{2[A K]}=f(K) d K
$$

und damit nach Gleichung 2:

$$
\mathrm{d}[\mathrm{ak}]=\frac{[\mathrm{AK}]}{1+\mathrm{K}[\mathrm{b}]^{2}} \cdot \mathrm{f}(\mathrm{K}) \mathrm{dK}
$$


also:

$$
\frac{[\mathrm{ak}]}{[\mathrm{AK}]}=\int_{-\infty}^{+\infty} \frac{f(\mathrm{~K})}{1+K[\mathrm{~h}]^{2}} \mathrm{dK} .
$$

Mit $B=2\left[h_{2} a k\right]$ ist dann

$$
1-\frac{B}{2[A K]}=\int_{-\infty}^{+\infty} \frac{f(K)}{1+K[h]^{2}} d K .
$$

Wählt man jetzt nach SIrs (13) $f(K)$ so, daß

$$
1-\int_{-\infty}^{+\infty} \frac{f(K)}{1+K x} d K=\frac{K x^{v}}{1+K x^{v}}
$$

gilt mit einem Heterogenitätsindex $\mathrm{V} \quad 0 \leqq \mathrm{~V} \leqq 1$, so gilt

$$
\frac{\mathrm{B}}{2[\mathrm{AK}]}=\frac{\mathrm{K}[\mathrm{h}]^{\mathrm{eV}}}{1+\mathrm{K}[\mathrm{h}]^{2 \mathrm{~V}}} .
$$

Speziell für $\mathrm{V}=1 / 2$ erhält man einen linearen $\mathrm{Zu}-$ sammenhang $z$ wischen $1 / B$ und $1 / F$. Heterogenitāt und Kooperativität können sich also in der Weise überlagern, da $B$ man allein aufgrund der Gleichgewichtsdaten eine Beschreibung der Hormon-Antikörperreaktion nach dem Schema:

$$
\mathrm{H}+\mathrm{AK} \rightleftharpoons \mathrm{HAK}
$$

nicht ausschließen kann.

\section{Experimentelle Untersuchungen}

${ }^{125} \mathrm{~J}-\mathrm{T}_{3}$-Bindungsversucbe (vgl. l. c. 4)

${ }^{125} \mathrm{~J}-\mathrm{T}_{3}$-Bindungsversuche wurden bei verschiedenen $\mathrm{pH}$-Werten, verschiedenen Inkubationstemperaturen und bei verschiedenen Antikörperpopulationen durchgeführt nach folgendem Schema: Inkubationsansatz:

1. ${ }^{125} \mathrm{~J}-\mathrm{T}_{3}$ (Byk-Mallinckrodt, Prankfurt), spezifische Aktivität etwa $100 \mathrm{mCi} / \mathrm{mg}$;

2. $50 \mu \mathrm{l}$ Antiserum (Endverdünnung 1:10000), Herstellung der Antisera nach 1. c. (14);

3. $0,05 \mathrm{~mol} / 1$ Tris-Puffer $\mathrm{pH} 8,0$ (bzw. $\mathrm{pH} 6,75, \mathrm{pH} 9,0$ ) mit 2,5 g/l Humanserumalbumin.

Ansteigende ${ }^{125} \mathrm{~J}-\mathrm{T}_{3}$ Konzentrationen wurden mit einer konstanten Menge Antiserum bei einem Endvolumen von $500 \mu \mathrm{l}$ über $12 \mathrm{~h}$ bei $4^{\circ} \mathrm{C}$ inkubiert. Die Wärmeinkubation exfolgte bei $37^{\circ} \mathrm{C}$ über $12 \mathrm{~h}$ in einem temperaturkonstanten Inkubator. Die Trennung von gebundenem und freiem markierten Hormon exfolgte durch Zugabe von Dextran-gesättigter Holzkohle und anschließendes Zentrifugieren (10 $\mathrm{min}, 2000 \mathrm{U} / \mathrm{min}$ ).

Um den Heterogenitātsgrad der verwendeten Antikörperpopulation zu erhöhen, wurde bei einem Bindungsversuch das Antiserum des Kaninchens 34 mit denen der Kaninchen 35 und 36 zu gleichen Teilen gemischt. Die Endverdünnung des Gemisches wird wie die der Einzelsera 1:10000 gewählt.

HCT-Standardkurve (nach l. c. 15)

Menschliches Calcitonin wurde nach der Chloramin-T-Methode auf eine spezifische Aktivitāt von etwa $200 \mathrm{mCi} / \mathrm{mg}$ markiert und anschließend chromatographisch gereinigt (15). Der hier verwendete Antikörper wurde durch Immunisierung mit reinem HCT in einer Ziege gewonnen. Die Endverdünnung des Antiserums betrug 1:2000. Der Inkubationsansatz, die verwendeten Puffer sind früher beschrieben (15).

Bei der Erstellung der Standardkurve wurden ansteigende Mengen von Hormonstandard $(0,5 \mathrm{pg}-80 \mathrm{pg})$ und konstante Mengen ${ }^{125} \mathrm{~J}$-HCT (etwa $1000 \mathrm{Imp}$./min) mit einer konstanten Antikörperkonzentration über $72 \mathrm{~h}$ bei $4^{\circ} \mathrm{C}$ inkubiert. Die Trennung von freiem und gebundenem Hormon erfolgte durch Zugabe von Dextran-gesättigter Holzkohle und anschließendes Zentrifugieren (15 min bei $2500 \mathrm{U} / \mathrm{min}$ ).

\section{HPL-Standardkurve}

Eine HPL-Standardkurve wurde mit einem Kit der Firma Pharmacia (Phadebas HCS-Test) erstellt. $2 \mathrm{ng}{ }^{125} \mathrm{~J}$-HPL (spezifische Aktivität $30 \mathrm{nCi} / \mathrm{ng}$ ) und steigende Mlengen Hormonstandard $(0,5 \mathrm{~g}-8,0 \mathrm{~g})$ werden $60 \mathrm{~min}$ in Raumtemperatur mit Anti-HPLAntiserum inkubiert, die Trennung von freier und gebundener Phase erfolgt durch Zusatz ron Athanol zu den Reaktionsgefäßen und Zentrifugieren ( $2 \mathrm{~min}, 2000 \mathrm{U} / \mathrm{min}$ ).

Beim $T_{3}$ und beim HCT werden der freie, beim HPL der gebundene Anteil des markierten Hormons in einem GammaProbenwechsler (Tracerlab) gezählt.

\section{Ergebnisse und Diskussion}

$\mathrm{Da}$ bei weniger simplen mathematischen Modellen in der Regel eine Vielzahl von verschiedenen Parametern frei variiert werden können, um ein sogenanntes „curvefitting" der experimentellen Daten zu ermöglichen, ist die Frage nach der Richtigkeit der zugrunde gelegten Vorstellungen nur durch eine Mehrzahl von verschiedenartigen, voneinander unabhängigen Experimenten überprüfbar. Wenn theoretisch erwartete Ergebnisse sich experimentell nicht bestätigen lassen, wird das benutzte Modell, werden die gemachten Annahmen und Voraussetzungen die Wirklichkeit jedenfalls nicht adāquat beschreiben. In unserer speziellen Situation, bei dem hier betrachteten Modell, kann nun folgendes erwartet werden:

1. Bei der Strukturähnlichkeit der meisten Antikörpermoleküle vom Typ IgG wird das Auftreten von aufsteigenden B/F-Kurven das Normale und kein vereinzeltes Phänomen sein.

Dies hat sich inzwischen bei einer ganzen Reihe von Hormon-Antikörperreaktionen bestätigt.

2. Ansteigende B/F-Kurven werden unabhängig von der Art, wie freie und gebundene Phase getrennt werden, auftreten.

Ansteigende B/F-Kurven haben sich nicht nur bei Adsorptionsmethoden $(3,4)$, sondern auch bei elektrophoretischen Trennverfahren $(3,16)$, bei Ammoniumsulfat-Präzipitationen (3), bei Alkoholpräzipitation ${ }^{3}$ ) und bei Doppelantikörperfällmethoden ${ }^{3}$ ) ergeben.

3. Analog den klassischen Resultaten bei der $\mathrm{O}_{2}-$ Bindung an Hämoglobin werden die postulierten Konformationsānderungen der Antikörpermoleküle eine spezifische Temperatur- und $\mathrm{pH}$-Wertabhängigkeit zeigen.

Dies ist von uns bei unserem Trijodthyroninradioimmunoassay nachgeprüft worden (Abb. 2).

4. Bei monovalenten Antikörperfragmenten (Fab-Fragmente) sind keine ansteigenden B/F-Kurven zu erwarten.

Diese Erwartung ist experimentell von WEINTRAUB et al. ${ }^{1}$ ) bestätigt worden.

5. Eine stark ausgeprāgte Heterogenität der betreffenden Antikörperpopulation muß das erwartete Bindungs-

3) Eigene Beobachtungen bei dem erwähnten HPL-Kit der Firma Pharmacia. 


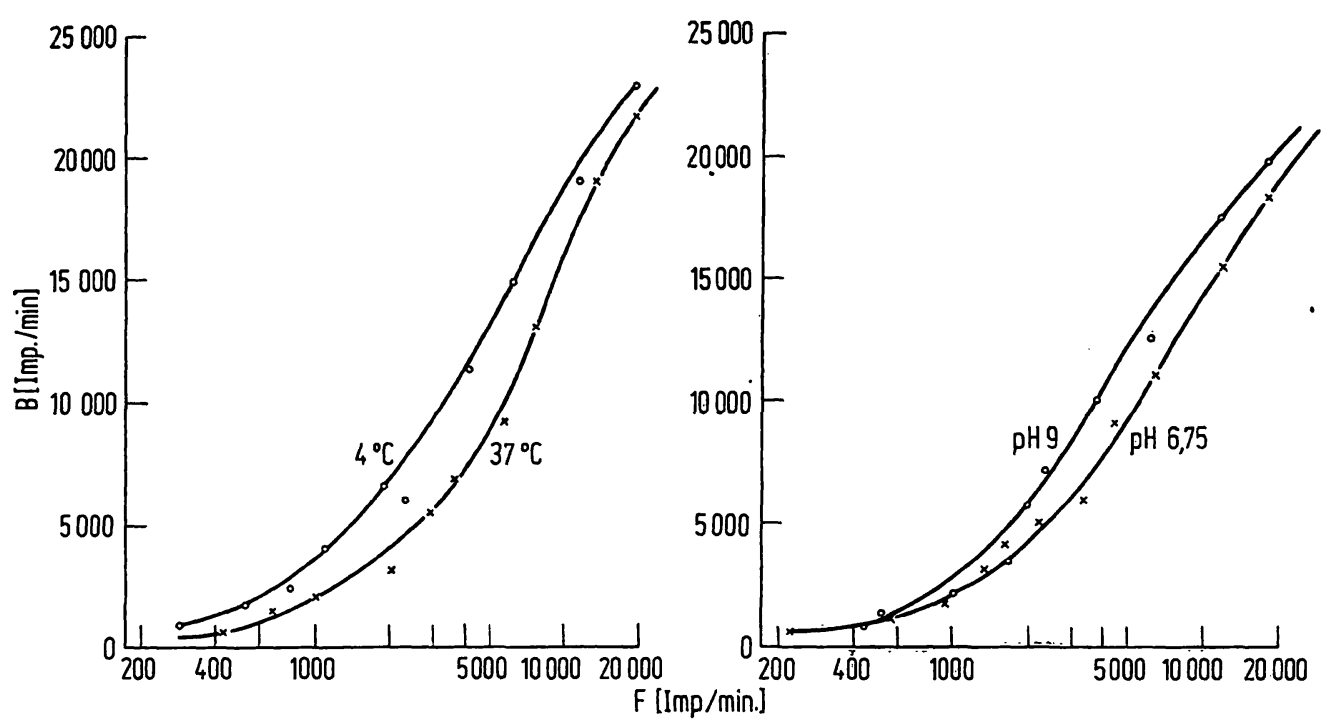

:

Abb. 2

$B$ als Funktion von $F$ bei ${ }^{12 s} \mathrm{~J}-\mathrm{Tri}-$ jodthyronin-Bindungsversuchen. Die halblogarithmische Auftragungsart vergrößert die nur schwach ausgeprägte Sigmoidität

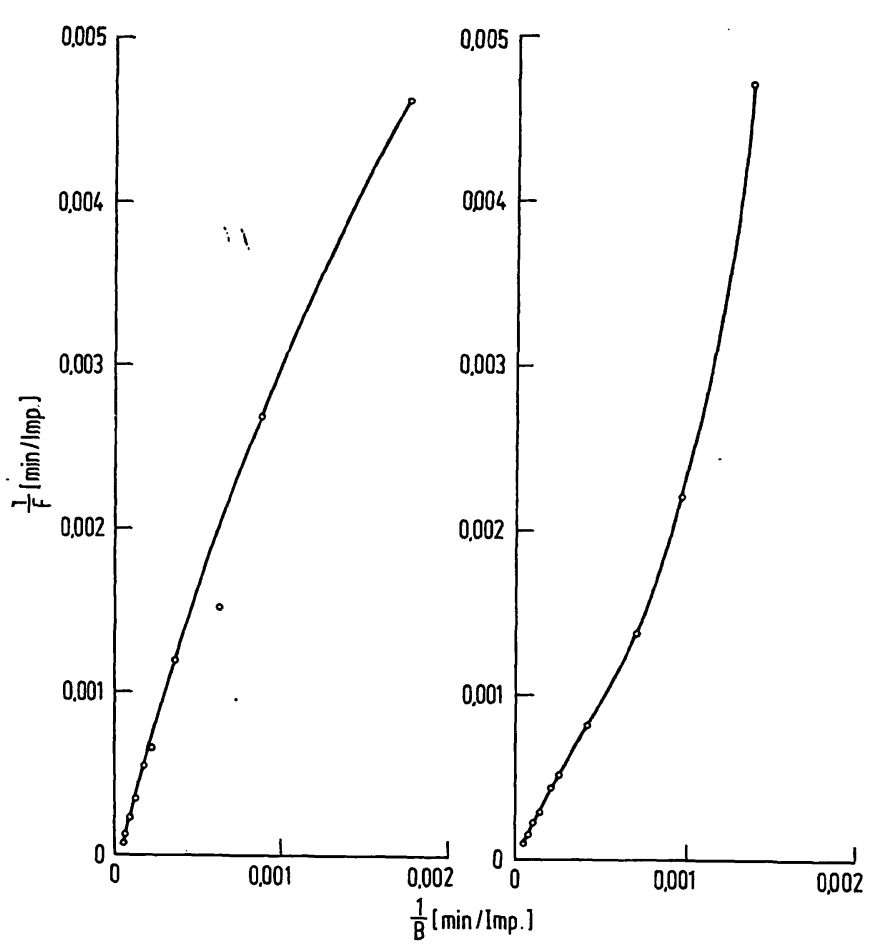

Abb. 3

1/F als Funktion von 1/B bei ${ }^{125} \mathrm{~J}$-Trijodthyronin-Bindungsversuchen. Bei der unvermischten Antikörperpopulation überwiegt deutlich die Kooperativität (links), bei dem Antikörpergemisch aus drei Populationen kommt die Heterogenität stärker zum Tragen (rechts)

verhalten entweder völlig überlagern oder wenigstens eine Abschwächung ergeben.

Der erwartete Überlagerungseffekt wird indirekt durch Bindungsstudien von Zimmering et al. (12) bei der Bindung von Testosteron-17-Hemisuccinat an spezifische Antikörper bestätigt. Nach Reinigung des Antiserums auf drei verschiedene Arten ergeben sich unterschiedliche Bindungsverhalten des gleichen Antikörpers, und zwar sigmoidale als auch hyperbolische Dissoziationskurven (1. c. 12, insbesondere S. 156). Das läßt sich durch verschiedene Heterogenitätsgrade der jeweiligen Antikörperpopulation plausibel erklären.

Umgekehrt liegt es nahe zu versuchen, den Heterogenitätsgrad von Antikörperpopulationen künstlich zu vergrößern: Nach Durchmischung von drei verschiedenen Antikörperpopulationen gegen $T_{3}$, die jede für sich noch Gleichgewichtskurven ergeben, die bei der Auftragung von $1 / \mathrm{F}$ gegenüber $1 / \mathrm{B}$ eine Krümmung in Richtung der 1/B-Achse aufweisen, ist dieses Bindungsverhalten durch die vergrößerte Heterogenität vollständig überlagert (Abb. 3).

6. Die Lage des maximalen B/F-Wertes wird die durch die Gleichungen 17, 18 und 19 ausgedrückte Antikörperkonzentrationsabhängigkeit zeigen.

Die erwähnten Gleichungen stimmen qualitativ mit den Ergebnissen von Matsukura (3) recht gut überein.

7. Bei Verwendung der sogeñannten ,temperaturejump"-Methode sollten für die verwendeten Antikörper vom Typ IgG zwei Relaxationseffekte nachweisbar sein (9).

Diese Frage ist vor einiger Zeit in Göttingen von A. Froese untersucht worden (17). Leider haben sich nur in wenigen Versuchsystemen bei der Messung der Relaxationszeiten Anhaltspunkte für eine Konformationsänderung der Antikörpermoleküle ergeben. Die ausgeprägte Heterogenität der betreffenden Antikörperpopulation macht sich auch hier störend bemerkbar, die bislang gefundenen Effekte sind schwach ausgeprägt und ermöglichen keine abschließende Entscheidung in der einen oder der anderen Weise (1. c. 17 S. 261/262). Ob bei den dort untersuchten AntigenAntikörperreaktionen gleichzeitig ansteigende $\mathrm{B} / \mathrm{F}$ Kurven beobachtet wurden, ist uns nicht bekannt.

8. Konformationsänderungen von Antikörpermolekülen sollteri auch zu heterotropen Effekten führen.

GrossBERG et al. berichteten 1965 von einem solchen Effekt: Nach der Bindung von Haptenen an spezifische Antikörper ist die enzymatische Proteolyse der Antikörpermoleküle durch Chymotrypsin deutlich reduziert (18).

Die von D. RoDBard kürzlich angeregte Beschreibung ${ }^{4}$ ) der Kooperativität bei der Bindung von Hormon-

4) Bislang unpublizierte Ergebnisse von D. RoDBARD, die uns aufgrund persönlicher Kommunikation bekannt sind. 

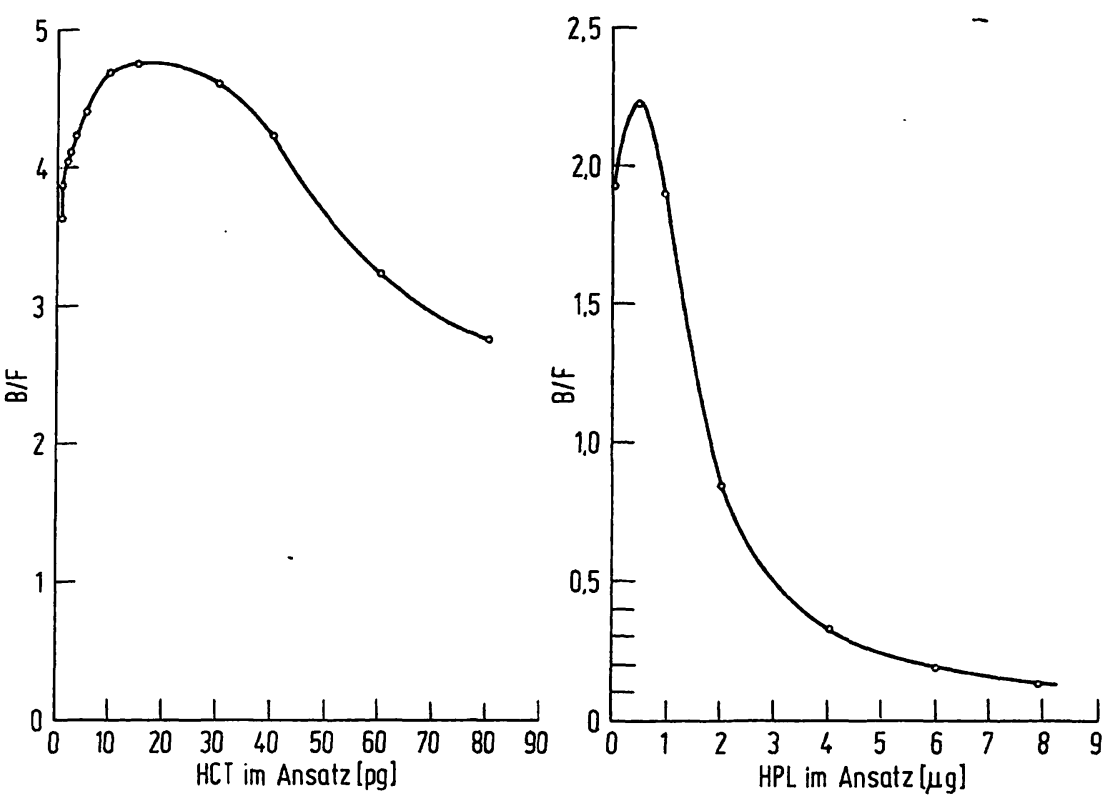

Abb. 4

Ansteigende B/F-Standardkurven beim Radioimmunassay von menschlichem Calcitonin (HCT) und menschJichem Plazenta-Laktationshormon (HPL) Kleinste , noch signifikant von Null unterscheidbare HCT im Ansatz [pg] Calcitonin-Konzentration: 1 pg

molekülen an Antikörper, die von gekoppelten Gleichgewichten

$$
\begin{gathered}
\mathrm{H}+\mathrm{AK} \rightleftharpoons \mathrm{HAK} \\
\mathrm{H}+\mathrm{HAK} \rightleftharpoons \mathrm{H}_{2} \mathrm{AK}
\end{gathered}
$$

mit Affinitätskonstanten $\mathrm{K}_{2} \gg \mathrm{K}_{1}$ ausgeht, ermöglicht keine quantitative Beschreibung von solchen heterotropen Effekten, kann also nicht alle bekannten experimentellen Daten beschreiben.

Die hier unter Punkt 5 aufgeführten experimentellen Ergebnisse gestatten noch eine wichtige Folgerung: $\mathrm{Da}$ die meisten Antigene mehrere Bindungsstellen gegenüber spezifischen Antikörpern besitzen, liegt es nicht fern, ausgehend von multivalenten Antigenen und mindestens bivalenten Antikörpern aufsteigende B/FKurven als Ergebnis von Präzipitationen anzusehen (Netzwerktheorie). Der maximale B/F-Wert befände sich dann in der sogenannten Äquivalenzzone, in einem Bereich optimaler Vernetzung. Der Einfluß einer wesentlichen Heterogenität der betreffenden Antikörperpopulation auf einen solchen Vernetzungsproze $B$ ist quantitativ von Pauling et al. 1944 diskutiert worden (19): Das Maximum in der Äquivalenzzone läßt sich $z$ war weniger scharf abgrenzen, wird aber nicht vollständig aufgehoben. Das hier nur durch Vergrößerung des Heterogenitätsgrades der Anti- $T_{3}$ Antikörperpopulation vollständig veränderte Bindungsverhalten (Abb. 3) scheint eine Erklärung aufsteigender B/F-Kurven mit Netzwerken auszuschließen.

Auf einen Gesichtspunkt sollte hicr allerdings noch eingegangen werden:

So wie auch bei einigen Enzym-Substrat-Reaktionen die Annahme von Konformationsänderungen keineswegs unzweifelhaft ist (20), sind auch bei Hormon-Antikörperreaktionen andere Mechanismen kooperativer Bindung denkbar: Die von Fisher-Gates-Cross angeregte Erklärung kooperativer Effekte läßt sich im Prinzip auch auf Antigen-Antikörperreaktionen übertragen. Immerhin, aufsteigende $\mathrm{B} / \mathrm{F}-\mathrm{Kurven}$ sind kein paradoxes Bindungsphänomen und die verfügbaren
Daten lassen eine Diskussion in der vorgenommenen Weise gerechtfertigt erscheinen.

Der an sich bekannte (l. c. 21) Zusammenhang zwischen ansteigenden B/F-Kurven und homotropen allosterischen Effekten ist im Bereich der klinisch orientierten Hormonforschung bislang wenig beachtet worden. Die bisher gefundenen Ergebnisse zur Charakterisierung optimaler Versuchsbedingungen bei radioimmunchemischen Hormonnachweismethoden gehen von zu einfachen Modellen der Hormon-Antikörperreaktion aus und sind in konkreten Fällen irreführend. Darauf haben wir kürzlich hingewiesen (4). Eine Vergrößerung der Empfindlichkeit ist uns in unserem Labor bei verschiedenen Versuchssystemen dadurch gelungen, da $\beta$ nach experimenteller Bestimmung von $[\mathrm{H}]_{\mathrm{MAX}}$ bei der jeweils benutzten Antikörperkonzentration die zugesetzte Menge markierten Hormons etwas größer als $[\mathrm{H}]_{\operatorname{Max}}$ gewählt wurde. Der Versuch, bei einem Calcitoninradioimmunoassay im aufsteigenden Teil der B/F-Kurven Hormonkonzentrationen $z u$ messen und damit eine Vergrößerung der Empfindlichkeit zu erreichen, hat sich deswegen als schwierig erwiesen, weil durch die relativ geringe spezifische Aktivität der markierten Calcitonin-Moleküle und bei der notwendig kleinen konstant zugesetzten Menge markierten Hormons zu geringe Zählraten eine hohe Streuung bewirken (vgl. Abb. 4).

Die naheliegende Frage nach ' der biologischen Bedeutung einer kooperativen Bindung von Antigenmolekülen an spezifische Antikörper und der Beeinflußbarkeit dieser Kooperativität durch Temperaturund $\mathrm{pH}$-Wertänderungen ist noch unklar und muß weiter untersucht werden.

\section{Danksagung}

Durch zahlreiche Literaturhinweise und helfende Kritik ist Herr Prof. Dr. H.-D. SöLING wesentlich an dem Zustandekommen dieser Arbeit beteiligt. Ihm sei deshalb an dieser Stelle ganz herzlich gedankt.

Ebenso sind die Autoren Frl. L. D. Frscher für das Schreiben des Manuskriptes zu Dank verpflichtet. 


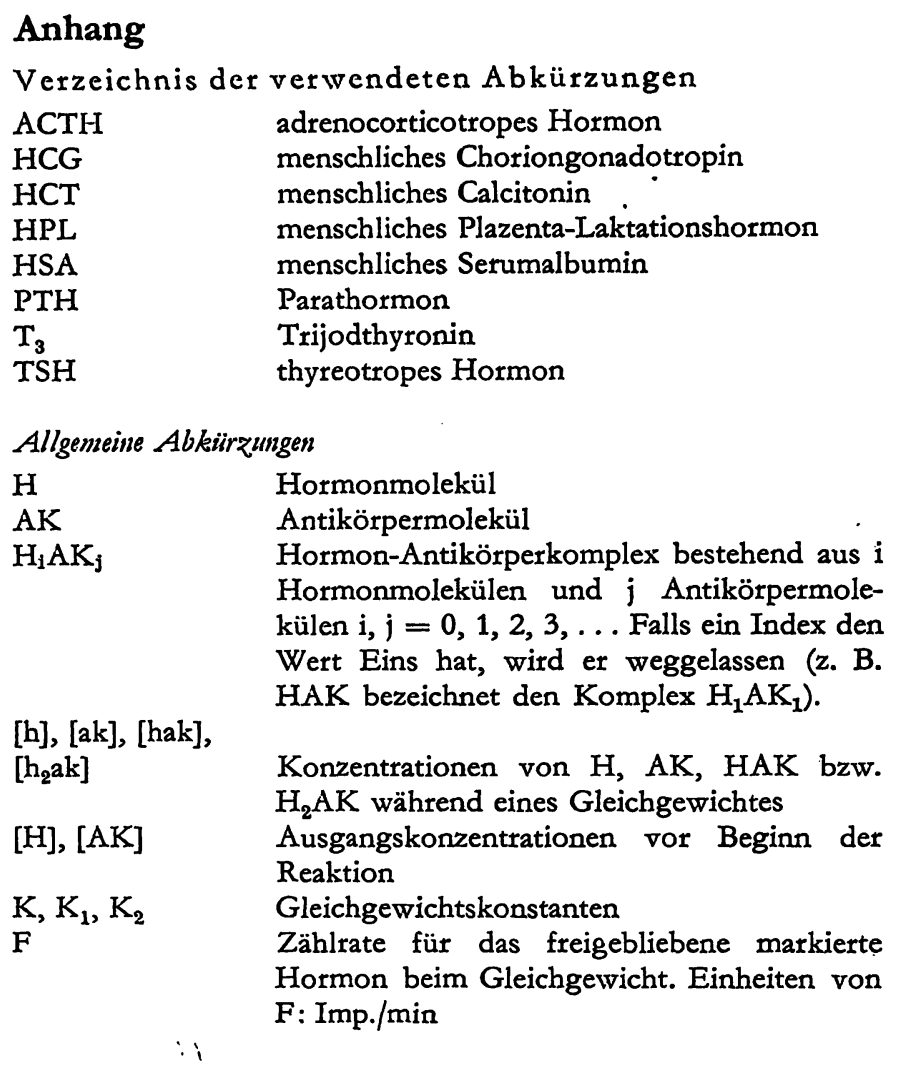

B

Ableürzungen
$\mathrm{R}_{\mathbf{1}}, \mathrm{T}_{\mathbf{1}}$
$\mathrm{L}$
$\mathrm{K}_{\mathrm{R}}, \mathrm{K}_{\mathrm{T}}$
$\mathrm{c}=\mathrm{K}_{\mathrm{R}} / \mathrm{K}_{\mathbf{T}}$
$\alpha=[\mathrm{h}] / \mathrm{K}_{\mathbf{R}}$
$\alpha_{\mathrm{MAX}}$

\section{Literatur}

1. Koshland, D. E. jr. \& Filmer, D. (1966), Biochemistry 5, 365-385. - 2. Monod, J., Wraran, J. \& Changeux, J.-P. (1965), J. Mol. Biol. 12, 88-118. - 3. Matsukura, S., West, C. D., Ichikawa, Y., Jubiz, W., Harada, G. \& TYleR, F. H. (1971), J. Lab. Clin. Med. 77, 490-500. - 4. Bartels, H.-J., Hesch, R. D. \& HÜFNER, M. (1972), diese Z. 10, 351-354. - 5. WeINtraub, B. D., KADESKy, Y. M. \& Rosen, S. W. (1972), Clin. Res. 20, 444. - 6. Klotz, I. M. (1953), in: The Proteins (NeUrath, H. \& Batley, K. eds.) Vol. I, Part B, 727-806, Acad. Press, New York. - 7. Berson, S. A. \& YaLow, R. S. (1959), J. Clin. Invest. 38, 1996-2016. - 8. Koshland, D. E. (1969), in: Current Topics in Cellular Regulation (HORECKER, B. L. \& Stadtaran, E. R. eds.) Vol. 1, 1-27, Acad. Pr., New York. 9. Kirschner, K., Eigen, M., Bitrman, R. \& Voigt, B. (1969), Proc. Nat. Acad. Sci., USA 56, 1661-1667. - 10. Porter, R. R.
$(B / F)_{\text {MAX }}$

$[\mathrm{H}]_{\text {MAX }}$

$\mathrm{V}$

Zählrate für das gebundene Hormon beim Gleichgewicht maximaler B/F-Wert einer Bindungs- bzw. Standardkurve bei fester Antikörperkonzentration

Ausgangshormonkonzentration, die bei fester Ausgangsantikörperkonzentration den maximalen B/F-Wert ergibt

duroh Verteilungsfunktion $f(K)$ bestimmter Heterogenitätsindex der betreffenden Antikörperpopulation', $0 \leqq \mathrm{~V} \leqq 1$.

Abkürrungen des allosterischen Modells (l. c. 2)

bezeichnen einmal die Komplexe $\mathrm{H}_{\mathrm{i}} \mathrm{AK}$, wobei sich das Antikörpermolekül im Rbzw. T-Zustand befindet, zum anderen aber auch die sich beim Gleichgewicht einstellenden Konzentrationen der betreffenden Komplexe Gleichgewichtskonstante zwischen $R$ - und $T-Z u s t a n d$ in Abwesenheit der Liganden Gleichgewichtskonstanten bei der HormonAntikörperbindung ' im R- bzw. T-Zustand

definiert durch $(\mathrm{B} / \mathrm{F})_{\mathrm{MAX}}=(\mathrm{B} / \mathrm{F})\left(\alpha_{\mathrm{MAX}}\right)$ bei fester Antikörperausgangskonzentration.

(1967), Sci. Amer. 217, 81-90. - 11. Nișsonoff, A. \& PressMAN, D. (1958), J. Immunol. 80, 417-428. - 12. ZimMERING, P. E., LIEBerman, S. \& Ertanger, B. F. (1967), Biochemistry 6, 154-164. - 13. Sips, R. (1948), J. Chem. Phys. 16, 490-495. 14. Hescr, R. D. \& HüFNER, M. (1972), Acta Biol. Med. Ger. 28, 861-864. - 15. HÜFNER, M. \& HESCH, R.-D. (1971), Klin. Wochenschr. 49, 1149-1150. - 16. The Wilson Laboratories, Bull. No. 23, Chicago, December 7 (1971). - 17. Froese, A. (1968), Immunochemistry 5, 253-264. - 18. Grossberg, A. L., Markus, G. \& Pressman, D. (1965), Proc. Nat. Acad. Sci., USA 54, 942-945. - 19. Pauling, L., Pressman, D. \& Grossberg, A. L. (1944), J. Amer. Chem. Soc. 66, 784-792. - 20. Frsher, H. F., Gates, R. E. \& CRoss, D. G. (1970), Nature (London) 228, 247-249. - 21. BlaNGy, D. (1971), Biochimie 53, 135-144.

Hans-Jochen Bartels Math. İnst. d. Univ. 34 Göttingen

Bunsenstr. 3/5 